\title{
Escala de evaluación del funcionamiento familiar FACES III: ¿Modelo de dos o tres factores?
}

\section{Family Functioning Evaluation Scale FACES III: Model of two or three factors?}

\author{
Vanina Schmidt, Juan Pablo Barreyro, Ana Laura Maglio \\ Consejo Nacional de Investigaciones Científicas y Técnicas (CONICET) Universidad de Buenos Aires (U.B.A.) \\ Disponible online 30 de abril de 2010
}

\begin{abstract}
La Escala de Evaluación de la Cohesión y Adaptabilidad Familiar (FACES III) de Olson, Portner, y Lavee, es una de las escalas desarrolladas para evaluar dos de las dimensiones del Modelo Circumplejo de Sistemas familiares y Maritales: la cohesión y la flexibilidad familiar. El propósito del presente estudio es contribuir a una mayor claridad respecto de los aspectos del funcionamiento familiar susceptibles de ser abordados con FACES III, así como proporcionar información sobre la validez estructural de la escala para su uso en población argentina. Participaron 785 padres $(M=41 ; D T=5.8)$ y 600 adolescentes $(M=16.3, D T=1.7)$ de la Ciudad Autónoma de Buenos Aires y Gran Buenos Aires a quienes se les administró el instrumento. Se realizó un análisis factorial confirmatorio. Los resultados indican que una estructura de dos factores no resulta del todo adecuada, mientras que un modelo de tres factores - Cohesión, Flexibilidad 1 y Flexibilidad 2 - presenta un buen ajuste a los datos. La dimensión Flexibilidad parece aquella con la que se debe ser más cauteloso teniendo en cuenta que probablemente esté reuniendo al menos dos constructos interconectados. Los resultados del presente estudio se discuten considerando los alcanzados en otros países con las distintas versiones de la escala.
\end{abstract}

Palabras Clave: Funcionamiento Familiar - FACES III - Análisis Factorial.

The Family Adaptability and Cohesion Evaluation Scale (FACES III) by Olson, Portner and Lavee was developed to assess two of Circumplex Model of Marital and Family Systems dimensions: the family cohesion and flexibility. The aim of this research is to contribute to determine the family functioning dimensions assessed by this instrument and to provide information about the structural validity of the scale for its application in Argentina population. Seven hundred and eighty-five parents $(M=41 ; S D=5.8)$ and six-hundred adolescents $(M=16.3, S D=1.7)$ from the City of Buenos Aires and Gran Buenos Aires participated in this study. The results showed that a two factor structure is not completely accurate while a three factor model -Cohesion, Flexibility 1 and Flexibility 2 - fits data well. According to these results, the dimension Flexibility is probably composed of, at least, two interconnected constructs. Results from the present research are discussed considering previous evidence obtained in other countries with different versions of the scale.

Keywords: Family Functioning - FACES III - Factor Analysis

\footnotetext{
Esta investigación ha sido financiada por el Consejo Nacional de Investigaciones Científicas y Técnicas (CONICET) a través del Proyecto "La comunicación del adolescente con sus padres como factor protector de abuso de alcohol. Interacción con variables disposicionales" (inserto en Proyecto UBACyT P-021).

Correspondencia: Dra. Vanina Schmidt, Pasaje Columbia 4934 (1417), Ciudad Autónoma de Buenos Aires - República Argentina. Telf. particular: 054-011-4639-8858, Fax: 054-011- 4957-5886, E-mail: vschmidt@psi.uba.ar
} 
La familia es uno de los contextos más relevantes en la vida del ser humano. Los estudios muestran el estrecho vínculo entre las experiencias vividas en la familia y la salud y el desarrollo del individuo (Arnett, 2008; Estévez-López, Murgui-Pérez, Moreno-Ruiz y Musitu-Ochoa, 2007; Lalueza y Crespo, 2003).

A partir de la década del 50 proliferaron los modelos de familia que intentan describir los patrones de interacción que ocurren en el sistema familiar (Beavers, y Hampson, 1995; Doherty y Colangelo, 1984; Epstein, Bishop y Baldwin, 1983; Minuchin, 1992; Olson, Russell,y Sprenkle, 1989; Steinhauer, Santa-Barbara, y Skinner, 1984). Existe actualmente un mayor consenso respecto de cuáles son los aspectos que deberían considerarse en la indagación del funcionamiento familiar.

Desde el Modelo Circumplejo de Sistemas familiares y Maritales (Olson, 2000; Olson, Russell y Sprenkle, 1989), el cual ha tenido una gran difusión en los últimos años en el mundo académico y profesional, se sostiene que la cohesión, la flexibilidad y la comunicación son las tres dimensiones que principalmente definen el constructo funcionamiento familiar. La cohesión se refiere al grado de unión emocional percibido por los miembros de la familia. La Flexibilidad familiar se define como la magnitud de cambio en roles, reglas y liderazgo que experimenta la familia. El grado de cohesión y flexibilidad que presenta cada familia puede constituir un indicador del tipo de funcionamiento que predomina en el sistema: extremo, de rango medio o balanceado (ver Tabla 1). Los sistemas maritales o familiares balanceados tienden a ser más funcionales y facilitadores del funcionamiento, siendo los extremos más problemáticos mientras la familia atraviesa el ciclo vital.

La comunicación familiar es la tercera dimensión del modelo y facilita el movimiento dentro de las otras dos dimensiones. Dado que en este artículo se presenta el resultado de la aplicación del instrumento que operacionaliza los dos primeros constructos, no nos ocuparemos aquí de la tercera dimensión del modelo medida con escalas especialmente diseñadas para evaluarla.

Tabla 1. Caracterización de los sistemas familiares y maritales según el nivel de cohesión y flexibilidad.

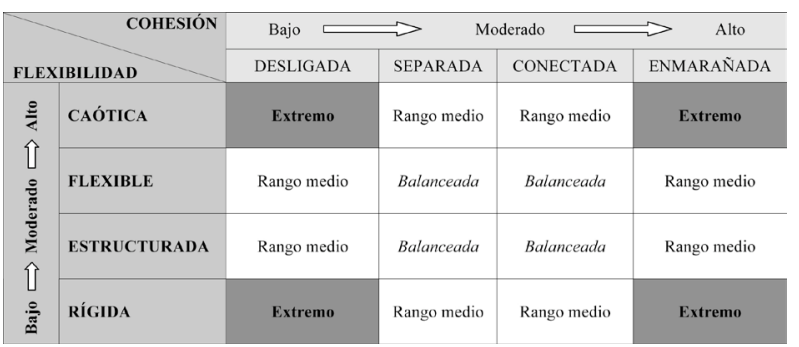

\section{La evaluación del funcionamiento familiar}

Evaluar el funcionamiento familiar a través de procedimientos rigurosos se ha convertido en una necesidad real de la práctica psicológica. Se utilizan actualmente una gran variedad de instrumentos para evaluar la familia a nivel de grupo (con- siderando el sistema familiar como un todo) (ver Tabla 2). La gran mayoría de los instrumentos están validados para ser utilizados en el contexto en el cual se han originado, pero no han sido adaptados a otros contextos.

Tabla 2. Instrumentos de evaluación del funcionamiento familiar más utilizados.

\begin{tabular}{|c|c|}
\hline Instrumento de evaluación & Autor/es y año de publicación \\
\hline $\begin{array}{l}\text { Escalas de Cohesión y Adaptabilidad } \\
\text { Familiar (FACES I, II, III y IV) }\end{array}$ & $\begin{array}{l}\text { Olson (1985) } \\
\text { Olson y Gorall (2003) }\end{array}$ \\
\hline $\begin{array}{l}\text { Escala de Comunicación Padres- } \\
\text { Adolescente (CAPS) }\end{array}$ & Barnes y Olson (1982) \\
\hline Escala de Puntuación Clínica (CRS) & Thomas y Olson (1993) \\
\hline $\begin{array}{l}\text { Recursos de Evaluación Familiar } \\
\text { (FAD) }\end{array}$ & Epstein, Bishop y Baldwin (1993) \\
\hline $\begin{array}{l}\text { Inventario Familiar de Autoreporte } \\
\text { (SFI) }\end{array}$ & Beavers, Hampson y Hughs (1985) \\
\hline Medida de Evaluación Familiar (FAM) & $\begin{array}{l}\text { Skinner, Steinhauer y Santa-Barba- } \\
\text { ra, (1983) }\end{array}$ \\
\hline Escala de Clima Familiar (FES) & Moos y Moos (1984) \\
\hline $\begin{array}{l}\text { Índice de Funcionamiento Familiar } \\
(F F I)\end{array}$ & Pless y Satterwhite (1973) \\
\hline $\begin{array}{l}\text { Índice de Funcionamiento Familiar } \\
(F F I)\end{array}$ & $\begin{array}{l}\text { Linder-Pelz, Levy, Tamir, Spencer y } \\
\text { Epstein (1984) }\end{array}$ \\
\hline$A P G A R$ Familiar & Smilkstein (1978) \\
\hline $\begin{array}{l}\text { Escala McMaster de valoración clínica } \\
(M C R S)\end{array}$ & $\begin{array}{l}\text { Miller, Ryan, Keitner, Bishop y } \\
\text { Epstein (2000) }\end{array}$ \\
\hline Escala de Adaptación Familiar (FAS) & Antonovsky y Sourani (1988) \\
\hline $\begin{array}{l}\text { Encuesta de Relación Padre-Hijo } \\
(P C R S)\end{array}$ & Fine y Schwebe (1983) \\
\hline Escala de Funcionamiento Familiar & Palomar (1998) \\
\hline Escala de Funcionamiento Familiar & $\begin{array}{l}\text { García Méndez, Rivera-Aragón, } \\
\text { Reyes-Lagunes y Díaz-Loving } \\
\text { (2006) }\end{array}$ \\
\hline
\end{tabular}

La replicabilidad transcultural de los constructos conlleva una serie de sesgos que deben ser advertidos y minimizados. En Psicología, en especial en Iberoamérica, se han tratado de minimizar tales sesgos generando modelos e instrumentos de familia culturalmente válidos.

La Escala de Evaluación de Cohesión y Adaptabilidad Familiar (FACES III; de Olson, Portner y Lavee, 1985; Olson, 1992) es la $3^{\text {o }}$ versión de la serie de escalas FACES y ha sido desarrollada para evaluar dos de las principales dimensiones del Modelo Circumplejo: la cohesión y la flexibilidad familiar. Se ha adaptado a una variedad de contextos culturales y se calcula que existen más de 700 estudios que utilizan FACES en sus distintas versiones (Kouneski, 2001; citado en Gorall, Tiesel y Olson, 2004). En la mayoría de los casos, la escala logra discriminar diferentes grupos familiares (por ejemplo, clínico y no clínico), lo cual constituye una fuerte evidencia de validez de constructo de la misma. Otra de las cualidades métricas que destacan los autores es que con FACES III se ha logrado bajar la correlación que existía entre ambos factores (cohesión y flexibilidad) cuando se utilizaba FACES II, y la correlación entre ellos con deseabilidad social. Además, el Análisis Factorial mostró que los ítems saturaban en el factor postulado (Olson, 1986, 1994; Olson, Portner y Lavee, 1985). 
En las sucesivas adaptaciones de la prueba se han obtenido resultados dispares en términos de evidencias de validez y fiabilidad. En población española, se suelen hallar adecuados índices de consistencia interna para cohesión, pero bajos para flexibilidad (Forjaz, Martinez-Cano y Cervera-Enguix, 2002; Polaino-Lorente y Martínez-Cano, 1995), a pesar de lo cual en el primero de estos trabajos referenciados se logró replicar la estructura original de FACES III. En México, se concluye que la prueba es relativamente confiable y válida, realizando un análisis factorial confirmatorio y estableciendo los coeficientes de confiabilidad para cada factor (Ponce-Rosas, Gómez-Clavelina, Terán-Trillo, Irigoyen-Coria y LandgraveIbáñez, 2002). Sin embargo, presentan unos índices de ajuste para el modelo de dos factores que paradójicamente estarían señalando "problemas de ajuste". Hasui, Kishida y Kitamura (2004) también realizan un análisis factorial confirmatorio para FACES III con estudiantes universitarios japoneses y presentan un modelo de dos factores con buen ajuste, pero sólo si se eliminan varios ítems y se realizan cambios en el contenido de la prueba. De hecho, los cambios son tan radicales que finalmente en el Factor I aparecen ítems construidos originariamente para evaluar aspectos de la Flexibilidad Familiar (ítems 4, 8, 10, y 14), y en el Factor II sólo pudieron conservar tres ítems, uno de ellos (ítem 17) construido para evaluar la Cohesión Familiar. Algo similar ocurre en la versión chilena (Zegers, Larraín, Polaino-Lorente, Trapo y Diez, 2003). En Suiza, al realizar un análisis factorial confirmatorio, se concluyó que un modelo de dos factores puede ser adecuado si se eliminan algunos ítems (Vandeleur, Preisig, Fenton y Ferrero, 1999). Sin embargo, presentan criterios de ajuste inferiores a los considerados aceptables por la mayoría de los estudios.

En síntesis, no son pocos los estudios que evidencian dificultades para replicar la estructura de dos factores para FACES III. Incluso en EE.UU., país de origen de la prueba, ha resultado difícil hallar una estructura bifactorial y en algunos estudios (por ejemplo, Crowley, 1998) se concluye que un modelo de tres factores describe mejor la situación de FACES III

Dado estos resultados, el objetivo del presente estudio fue determinar la estructura interna de la escala FACES III en una muestra de Argentina.

Otros parámetros de calidad métrica ya habían sido indagados en nuestro contexto con resultados favorables (Leibovich y Schmidt, 2010; Maglio, 2003, 2005; Schmidt, 2001, 2002, 2003, 2007), convirtiéndose en una de las pruebas de evaluación familiar más utilizadas de nuestro medio.

Con la presente investigación se pretende contribuir a una mayor claridad respecto de los aspectos del funcionamiento familiar susceptibles de ser abordados con FACES III, así como proporcionar información sobre la validez estructural de la escala para su uso en población argentina.
Método

\section{Participantes}

Participaron un total de 1385 sujetos de la Ciudad Autónoma de Buenos Aires y Gran Buenos Aires ( $1^{\circ}$ cordón). Un subgrupo de ellos $(n=785)$ eran madre o padre de al menos un hijo con el cual convivían. Las edades de los hijos iban de 3 meses a 25 años. La mayoría de los padres (85\%) estaba casado o convivía con una pareja, el $10 \%$ era separado, el 4 $\%$ soltero y el $1 \%$ viudo. El $20 \%$ de los padres, tenía hasta educación secundaria incompleta; el $25 \%$ había realizado la educación secundaria completa y el resto, había cursado educación superior o universitaria completa o incompleta. El 15\% de estos hogares estaban constituidos por un hijo; el $46 \%$, de dos hijos; el $29 \%$, de tres hijos y, el resto (10\%), entre cuatro y seis hijos. El $55 \%$ de los hogares, tenía hijos menores de doce años. Participaron el $62 \%$ de las madres, quienes tenían una edad promedio de 41 años $(D T=5.8)$ y el $48 \%$ de los padres, que tenían una edad promedio de 40 años $(D T=6)$.

A su vez, colaboraron con el presente estudio 600 hijos adolescentes entre 12 y 25 años $(M=16.3, D T=1.7)$ previo consentimiento informado por escrito por parte de los padres cuando se trató de sujetos menores de 18 años. El $38 \%$ era varón y el 95\% estaba cursando estudios secundarios.

\section{Instrumentos}

Cuestionario socio-demográfico y socio-familiar construido ad hoc. Permitió obtener datos acerca de las características sociodemográficas de los participantes.

Escala de Evaluación de la Cohesión y Adaptabilidad Familiar, $3^{\circ}$ Versión (FACES III) de Olson, Portner y Lavee (1985) y Olson (1992), en la adaptación de Zamponi y Pereyra (1997) y Schmidt (2002, 2003; Leibovich y Schmidt, 2010). Consta de 40 ítems, cada uno con una escala Likert de cinco opciones (casi siempre, muchas veces, a veces sí y a veces no, pocas veces, casi nunca), divididos en dos partes. La parte I, compuesta por 20 ítems y que evalúa el nivel de cohesión y flexibilidad de la familia tal como el sujeto la percibe en ese momento ("Familia Real"). Y la parte II, compuesta por 20 ítems que reflejan el nivel de cohesión y flexibilidad que al sujeto le gustaría que hubiese en su familia ("Familia Ideal"). Esta parte incluye los mismos ítems que primera, pero se modifica la consigna general. De las diferencias entre las escalas "real" e "ideal" se puede obtener el índice de satisfacción que la persona tiene con el funcionamiento de su familia. Dados los fines del presente estudio, sólo se trabajó con los datos de la "Familia Real".

\section{Análisis de datos}

Para identificar cuáles son los factores o dimensiones que subyacen a los ítems de FACES III se utilizó el Análisis Facto- 
rial Confirmatorio (AFC). Es un análisis adecuado para examinar la posible estructura o modelo en función de las variables latentes que subyacen a los ítems, y observar el nivel de ajuste de los modelos predichos a los datos. Este análisis se ha llevado a cabo utilizando la estimación de máxima verosimilitud entre los ítems como input para el análisis de datos (Arbuckle, 2003).

Respecto de los índices de ajuste, fueron seleccionados aquellos que surgieron siguiendo convenciones y recomendaciones (Hair, Anderson, Tathan y Black, 1998; Jaccard y Wan, 1996; Kline, 1998). Para los índices de ajustes basados en la comparación entre las covarianzas del modelo frente a las covarianzas observadas, se seleccionaron el índice AGFI (Adjusted Goodness of Fit Index) y el índice RMR (Root Mean Residual); el primero es una extensión de otro índice llamado GFI (Goodness of Fit Index) que representa el grado de ajuste conjunto del modelo sin tener en cuenta los grados de libertad. AGFI, en cambio, ajusta utilizando la razón obtenida entre los grados de libertad del modelo propuesto y los grados de libertad del modelo nulo (un nivel aceptable y recomendado es un valor mayor o igual a .90). $A G F I$ se considera un índice de ajuste $\mathrm{y}$, a la vez, de parsimonia ya que penaliza los modelos con muchos parámetros. El índice $R M R$, que se obtiene de calcular la raíz cuadrada de los residuos al cuadrado de la diferencia entre las matrices observadas y predichas, minimiza el sesgo producido por el tamaño de la muestra. Para este indicador se pueden considerar que valores iguales o inferiores a .08 indican un buen ajuste (Hair et al., 1998). Entre los índices basados en la comparación del modelo con un modelo alternativo, se ha seleccionado el índice CFI (Comparative Fit Index) y el índice IFI (Incremental Fit Index). CFI compara el ajuste entre la matriz de covarianzas que predice el modelo y la matriz de covarianzas observadas, con el ajuste de la matriz del modelo nulo y la matriz de covarianzas observadas. $C F I$ mide el porcentaje de pérdida que se produce en el ajuste, al cambiar del modelo predicho al modelo nulo. IFI, a diferencia del primero, es más consistente y tiene en cuenta los grados de libertad del modelo. Ambos índices deben ser superiores, por convención, a .90 (Shumacker y Lomax, 1996). Por último, entre los índices basados en las covarianzas del modelo frente a las observadas, corregidos por la pérdida de parsimonia, se ha seleccionado el índice RMSEA (Root Mean Square Error of Aproximation). El valor RMSEA representa el nivel de discrepancia entre el modelo y los datos en la población. Se consideran valores razonables de ajuste a la población aquellos inferiores a .08 (Browne y Cudeck, 1993).

\section{Resultados}

Con el objetivo de conocer cuáles son los factores o dimensiones que subyacen a los ítems de familia real de la escala FACES III se llevaron a cabo distintos procedimientos propios del análisis factorial confirmatorio. Se propusieron modelos de uno, dos y tres factores. De la escala general se omitieron los ítems 3, 5 y 7 de Cohesión y los ítems 16, 18 y 20 de Flexibilidad porque generaban una estructura total poco coherente.

En primer lugar, se realizó un análisis confirmatorio inicial de un factor sobre los ítems de familia real de la escala FACES III. Los índices de ajuste obtenidos mostraron, tal como se esperaba, que un modelo de un factor era insuficiente para poder explicar los resultados de las covarianzas observadas $(A G F I=.851 ; R M R=.102 ; C F I=.711 ; I F I=.713 ; R M S E A$ $=.078)$. Se realizó luego, un análisis confirmatorio de dos factores sobre los ítems (Fig. 1). Los resultados de los índices de ajuste del modelo a los datos mostraron que, a pesar de que el modelo presentó valores de ajuste mejores al modelo de un factor y en algunos índices buenos ajustes, el mismo resultó insuficiente para poder explicar los resultados de las varianzas y covarianzas observadas en la muestra $(A G F I=.913 ; R M R=$ $.085 ; C F I=.856 ; I F I=.857 ; R M S E A=.074)$. Por tal motivo, se llevó a cabo un tercer análisis con los ítems de la escala, proponiendo tres factores (ver Fig. 2). El modelo presentó el mejor ajuste a los datos, siendo superior en los índices a los modelos de uno y dos factores $(A G F I=.932 ; R M R=.07 ; C F I=.90 ; I F I$ $=.90 ; R M S E A=.063)$. Con el propósito de comparar el modelo de dos y de tres factores, se utilizó el sistema de comparación de modelos mediante la razón $Q$ de Jöreskog (1979). La razón $Q$ obtenida (Modelo de 2 factores $\chi^{2}=515.45 ; G L=75$; Modelo de 3 factores $\chi^{2}=478.76$; $G L=74 ; Q=36.69 ; p<.001$ ) indica que ambos modelos son significativamente diferentes. En concordancia con los resultados obtenidos, se puede afirmar que el modelo de tres factores tendría mayor capacidad predictiva que el modelo de dos factores.

Figura 1. Modelo de dos Factores. Pesos de Regresión y Correlación entre los Factores.

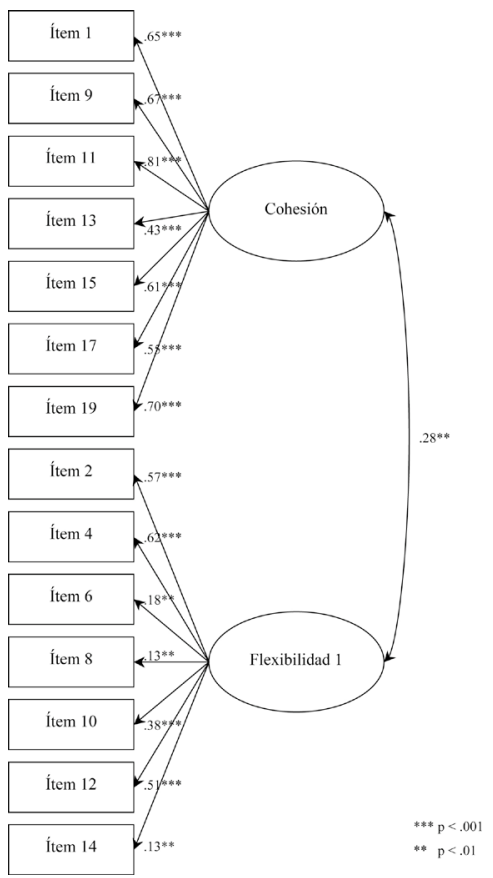


Figura 2. Modelo de tres Factores. Pesos de Regresión y Correlaciones entre los Factores.

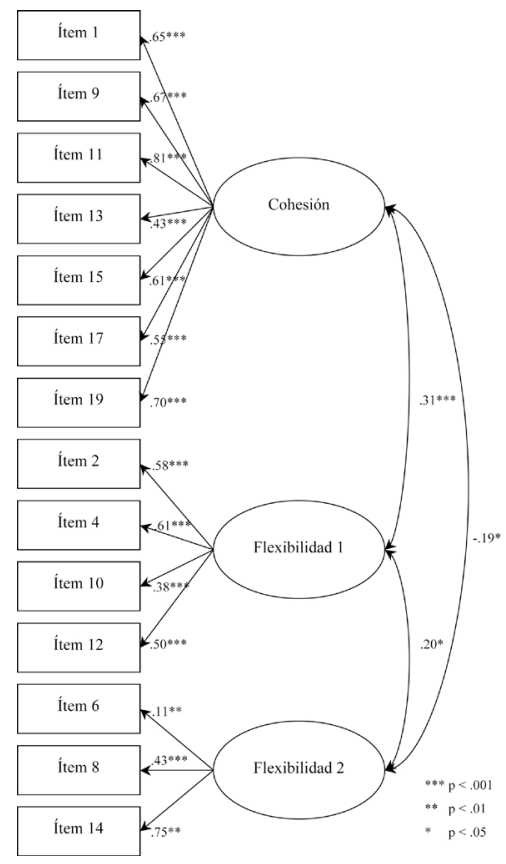

Respecto de los pesos de regresión del modelo de tres factores, se encontró que los mismos oscilaron entre .43 a .81 en el primer factor, .38 a .58 en el segundo factor y .11 a .75 en el tercer factor. Con relación a las correlaciones halladas, se observaron correlaciones positivas y significativas entre el factor I y II $(r=.31 ; p<.001)$, y entre el factor II y III $(r=.19, p$ $=.01)$, y correlaciones negativas y significativas entre el factor I y III $(r=-.19 ; p=.01)$.

La subescala de Cohesión mostró una elevada fiabilidad por consistencia interna $(\alpha=.82)$. La subescala Flexibilidad 1 mostró un grado de fiabilidad aceptable $(\alpha=.60)$; en cambio, la subescala Flexibilidad 2 mostró una fiabilidad baja $(\alpha=.37)$.

\section{Discusión}

En el presente trabajo se ha logrado obtener mayor información sobre la validez de constructo de FACES III a través del análisis factorial confirmatorio. Se observó que una estructura de dos factores no resulta del todo adecuada (si bien muestra mejor ajuste que un modelo de un factor), mientras que un modelo de tres factores presenta un buen ajuste a los datos.

Se obtiene de esta forma un primer factor con ítems referidos a la unión emocional (por ejemplo: Item 1. Los miembros de mi familia se dan apoyo entre si). En este factor, de hecho, cargan todos los ítems de la dimensión Cohesión de la versión original de FACES III. Los tres ítems eliminados (3, 5 y 7) por los motivos expuestos anteriormente, no hacen referencia directa al sentimiento de unión emocional sino que aluden a la relación de la familia con personas ajenas al sistema. El segundo factor obtenido queda compuesto por cuatro ítems que corresponden a la variable Flexibilidad de la versión original, los cuales aluden a la relación con los hijos en términos de disciplina y tipo de liderazgo que se ejerce sobre ellos (por ejemplo: Item 4. Los hijos también opinan sobre su disciplina). Se sugiere denominar a este factor, provisoriamente, Liderazgo parental. El tercer factor obtenido queda compuesto por tres ítems que aluden a la flexibilidad con respecto a las reglas y los roles familiares (por ejemplo: Item 14. En mi familia, las reglas suelen cambiar). Se sugiere denominar a este factor Reglas y Roles. Los ítems 16,18 y 20 de la variable Flexibilidad de la versión original de FACES III que fueron eliminados, se refieren a intercambios de quehaceres domésticos y funciones dentro del hogar.

En otras versiones, el $A F C$ ha logrado replicar el modelo de dos factores cuando se utilizan unos criterios de adecuación dato-modelo muy laxos (Ponce-Rosas et al., 2002; Vandeleur et al., 1999) o cuando se modifica radicalmente el contenido de cada variables (Hasui et al., 2004; Zegers et al., 2003), resultando una estructura poco coherente desde el punto de vista teórico. En síntesis, la evidencia disponible estaría señalando que los datos no se ajustan de modo adecuado a un modelo bifactorial. Ya Crowley (1998), en EE.UU., al realizar su $A F C$ había advertido que es necesario descartar el modelo de dos factores, habiendo obtenido un modelo de tres factores que es el de mejor ajuste. Presenta un factor de Cohesión y dos de flexibilidad: Disciplina y control percibido, que contiene los mismos ítems que Flexibilidad 1 del presente estudio y Flexibilidad percibida que contiene los ítems de Flexibilidad 2 del presente estudio, junto con los tres ítems que quedaron por fuera de nuestro modelo.

Los mayores problemas parecen estar del lado de la dimensión flexibilidad: la misma no se comporta como un constructo unitario. El problema con Flexibilidad no es nuevo pues, desde el punto de vista empírico, es la escala de peor calidad métrica: la de menor fiabilidad, la de más bajos saturaciones en los ítems que la componen y con una relación más oscura con otros criterios. En el manual de la prueba, se observa que para la variable cohesión pesos factoriales iban de .34 a .61 y para la variable flexibilidad, de .10 a .55 . Las saturaciones tan bajas en este factor son indicativas de problemas métricos, con lo que el problema se habría generado entonces en la construcción del instrumento.

Desde el punto de vista conceptual, nunca fue del todo clara esta dimensión ni siquiera para el propio autor. Solía decirse que Flexibilidad medía capacidad o habilidad de la familia para cambiar su estructura de liderazgo, roles y reglas en respuesta al estrés de desarrollo y cambios no normativos. Pero un análisis más profundo hizo concluir al autor que lo que se está midiendo con esta subescala es la magnitud de cambio y no la capacidad de cambio (Olson, 1994). Además, propuso reemplazar el término Adaptabilidad por el de Flexibilidad, por la confusión semántica que este último término generaba al asociarse fácilmente a otros (Olson, 1994). Pero no conforme con la claridad conceptual de Flexibilidad, a propósito de FACES 
IV, Olson revisa una vez más la definición de Flexibilidad y dice: "La Flexibilidad será definida ahora como la cualidad y expresión de liderazgo y organización, roles, reglas y negociaciones" (Gorall, Tiesel y Olson, 2004, p. 24).

Watson, Tiesel y Gorall (2004) concluyen que los resultados empíricos han modificado la teoría, y en vez de la dimensión flexibilidad, proponen tres aspectos del concepto relativamente independientes: resolución de conflictos, organización e inflexibilidad.

¿Debería desaconsejarse el uso de FACES III? Es fundamental considerar las diversas fuentes de validez disponibles a la hora de valorar la escala. La validez aparente, de contenido, relacionada con otros criterios, la utilidad demostrada de la prueba para discriminar diversos grupos familiares, orientar intervenciones, realizar evaluaciones, ha sido claramente establecida en trabajos nacionales e internacionales (Maglio, 2003; Olson, 2000; Schmidt, 2003, 2007).

Utilizar de modo responsable una escala implica conocer las limitaciones de la misma, pero no necesariamente descartar su uso. Hay una gran cantidad de evidencias del buen funcionamiento de la escala, a pesar de lo cual se debe tener presente que hay algunos ítems de ambos constructos que no parecen tan relevantes (ya que caen en el $A F C$ ), y la variable flexibilidad parece aquella con la que se debería ser más cautelosos teniendo en cuenta que probablemente esté reuniendo al menos dos constructos que, si bien correlacionan, lo hacen de forma muy leve.

Las buenas evaluaciones no dependen sólo de los buenos instrumentos. Se requiere profesionales adecuadamente capacitados en evaluación y en temas de familia, que lleven adelante el proceso de manera ética y responsable, adoptando una perspectiva multipersona, multimétodo, multirasgo y multisistema (Gorall, Tiesel y Olson, 2004), profesionales atentos a las posibilidades y limitaciones de las pruebas, pero también a los propios sesgos y prejuicios, que respeten la diversidad cultural, de género, y la elección individual de cómo vivir en familia, capaz de cuestionar las nociones vigentes de salud-enfermedad, funcionalidad-disfuncionalidad y normalidad-anormalidad.

\section{Nota de Autor}

Se agradece a las familias que participaron en la investigación, a la Licenciada Valeria Pedrón por sus aportaciones y al Instituto de Investigaciones de la Facultad de Psicología de la Universidad de Buenos Aires (sede del proyecto).

\section{Referencias}

Arbuckle, J. L. (2003). AMOS 5.0. Chicago, IL: SmallWaters

Arnett, J.J. (2008). Adolescencia y adultez emergente. Un enfoque cultural. Tercera edición. México: Pearson Prentice Hall.

Beavers, W. y Hampson, R. (1995). Familias exitosas. Eval- uación, tratamiento e intervención. Barcelona: Paidós.

Browne, M. W. y Cudeck, R. (1993). Alternative way of assessing model fit. En K. A. Bollen y J. S. Long (Eds.), Testing Structural Equation Models 8136-162). Newbury Park: Sage Publication

Crowley, S.L. (1998). A Psychometric Investigation of the FACES III: Confirmatory Factor Analysis with Replication. Early Education y Development, 9, 161-178.

Doherty, W. y Colangelo, N. (1984). The family FIRO model: A modest proposal for organizing family treatment. Journal of Marital and Family Therapy, 10, 19-29.

Epstein, N., Baldwin, L. y Bishop, D. (1983). The McMaster Model of Family Functioning: a view of the normal family. En F. Walsh (Ed), Normal family processes (pp. 115-142). NY: Guilford Press.

Estévez-López, E., Murgui-Pérez, S., Moreno-Ruiz, D. y Musitu-Ochoa, G. (2007). Estilos de comunicación familiar, actitud hacia la autoridad institucional y conducta violenta. Psicothema, 19, 108-113.

Forjaz, M.J., Martinez Cano, P. y Cervera-Enguix, S. (2002). Confirmatory factor analysis, reliability and validity of a Spanish version of FACES III. The American Journal of Family Therapy, 30, 439-449.

Gorall, D.M., Tiesel, J. y Olson, D.H. (2004). FACES IV Development and Validation. Minneapolis: Life Innovation.

Hair, F., Anderson, R. E, Tatham, R. L. y Black, W. C. (1998). Multivariate data analysis with readings. New Jersey: Prentice Hall.

Hasui, C., Kishida, Y. y Kitamura, T. (2004). Factor Structure of the FACES III in Japanese University Students. Family Process, 43, 33-140.

Jaccard, J. y Wan, C. K. (1996). LISREL approaches to interaction effects in multiple regression. Thousand Oaks, USA: Sage Publications.

Jöreskog, K.G. (1979). A general approach to confirmatory maximum likelihood factor analysis with addendum. En K.G. Jöreskog y D. Sörbom. Advances in factor analysis and structural equation models. Cambridge (MA): Abt Books.

Kline, R. B. (1998). Principles and practice of structural equation modeling. New York: Guilford Press.

Lalueza, J.L. y Crespo, I. (2003). Adolescencia y relaciones familiares. En A. Perinat (Comp.), Adolescentes del siglo XXI. Aproximación psicológica y social (1-21). Barcelona: EDIUOC.

Leibovich, N. y Schmidt, V. (2010). El contexto familiar y su evaluación desde una perspectiva ecopsicológica. Buenos Aires: Guadalupe.

Maglio, A. (2003). "La inseguridad laboral como estresor. La familia como recurso de afrontamiento y el impacto sobre el estado de ansiedad", Informe Beca Estímulo UBACyT. Buenos Aires: Facultad de Psicología, Universidad de Buenos Aires. 
Maglio A. (2005). Estudio del grupo familiar de pacientes con trastornos alimentarios: propuesta de un modelo conceptual y de evaluación para su abordaje. Memorias de las XII Jornadas de Investigación de la Facultad de Psicología de la Universidad de Buenos Aires, 1, 62-63.

Minuchin, S. (1992). Familia y terapia familiar. 4 ed. Barcelona: Gedisa.

Olson, D.H. (1986). Circumplex model VII: validation studies and FACES III, Familiy Process Journal, 25, 337-351.

Olson, D.H. (1994). Curvilinearity Survives: The World Is Not Flat, Family Process Journal, 33, 471-478.

Olson, D.H. (2000). Circumplex model of Marital and Family Systems, Journal of Family Therapy, 22, 144-167.

Olson, D.H., McCubbin, H.I., Barnes, H., Larsen, A., Muxen, M. y Wilson, M. (1992) Family Inventories. Minneapolis: Life Innovation.

Olson, D.H., Portner, J. y Lavee, Y. (1985). Manual de la Escala de Cohesión y Adaptabilidad Familiar (FACES III Manual). Minneapolis: Life Innovation.

Olson, D.H., Russell, C. y Sprenkle, D.H. (1989). Circumplex Model of Marital and Family Systems II: Empirical studies and clinical intervention. Advances in Family Intervention, Assessment and Theory, 1, 129-176.

Polaino-Lorente, A. y Martínez-Cano, P. (1995) El índice de fiabilidad de las "Familiy Adaptability and Cohesion Evaluation Scales" ( $3^{\circ}$ Versión), en una muestra de población española, Revista Psiquis, 16, 105-112.

Ponce-Rosas, E.R., Gómez-Clavelina, F.J., Terán-Trillo, M., Irigoyen-Coria, A.E. y Landgrave-Ibáñez, S. (2002). Validez de constructo del cuestionario FACES III en español (México). Atención primaria, 30, 624-630.

Schmidt, V. (2002). Escala de Evaluación de Cohesión y Adaptabilidad Familiar, $3^{\circ}$ versión (FACES III) y su uso en nuestro medio. Departamento de Publicaciones. Facultad de Psicología, U.B.A. Bs. As.

Schmidt, V. (2003). Estrés en familias de drogodependientes. Tesis de Doctorado. Buenos Aires: Facultad de Psicología, U.B.A.
Schmidt, V. (2007). Predictores de abuso de alcohol en adolescentes. Mitos versus evidencia empírica. XIV Anuario de Investigaciones, Año 2006. Facultad de Psicología, Universidad de Buenos Aires, Tomo I: 229-238.

Shumacker, R. E. y Lomax, R. G. (1996). A beginner guide to structural equation modeling. Mahwah: Erlbaum

Spijkerman, R., van den Eijnden, R.J. y Huiberts, A. (2008). Socioeconomic differences in alcohol-specific parenting practices and adolescents' drinking patterns. European Addiction Research, 14, 26-37.

Steinhauer, P., Santa-Barbara, J. y Skinner, H. (1984) The process model of family functioning. Canadian Journal of Psychiatry, 29, 77-88.

Vandeleur, C.L., Preisig, M., Fenton, B.T. y Ferrero, F. (1999). Construct validity and internal reliability of a French version of FACES III in adolescents and adults. Swiss Journal of Psychology, 58, 161-169.

Watson Tiesel, J. y Gorall, D. (2004). Agony or Ecstasy? Evolving theory and methods of the circumplex model. En: V. Bengston, A. Acock, D. Klein, K. Allen, K. y P. DilworthAnderson (eds). Sourcebook of family theory and research 8pp. 50-53). California: Sage.

Zamponi, J. y Pereyra, M. (1997) Validación de la Family Adaptability and Cohesion Evaluation Scales (FACES III) en una población argentina. Ficha de la Universidad Adventista del Plata.

Zegers, B., Larraín, M.E., Polaino-Lorente, A., Trapp, A. y Diez, I. (2003). Validez y confiabilidad de la versión española de la escala de Cohesión y Adaptabilidad Familiar (CAF) de Olson, Russell y Sprenkle para el diagnóstico del funcionamiento familiar en la población chilena. Revista chilena de neuro-psiquiatría, 41, 39-54.

Fecha de recepción: 6 de octubre de 2009 Fecha de aceptación: 17 de marzo de 2010 